Escuela de Ciencias Sociales y Humanidades, UNED, Costa Rica https://revistas.uned.ac.cr/index.php/espiga

ISSN: 1409-4002 • e-ISSN: 2215-454X

\title{
Propuesta metodológica de un estudio de caso sobre la educación a distancia en tiempos de pandemia
}

\section{Methodological Proposal about a Case Study on Distance Education in Pandemic Times}

Proposition d'une méthodologie pour une étude de cas sur l'enseignement à distance en temps de pandémie

Jonathan Elizondo-Mejías *

https://orcid.org/0000-0001-7559-5350

Patricia López-Estrada **

https://orcid.org/0000-0002-4811-5865

Estefanía Pérez-Hidalgo ***

https://orcid.org/0000-0003-3552-1781

Recibido: 15 de diciembre de 2020 Aceptado: 4 de febrero de 2021 
* Maestría en la Enseñanza del Inglés como Lengua Extranjera y Bachillerato en Ciencias de la Educación Primaria con Concentración en Inglés de la Universidad de Costa Rica, de Costa Rica. Bachillerato en la Enseñanza del Inglés de la Universidad Americana, de Costa Rica. Es profesortutor en la cátedra de Enseñanza del Inglés de la Escuela de Ciencias Sociales y Humanidades de la UNED y profesor de Lengua Extranjera en el Ministerio de Educación Pública. Correo: jonnathanelizondo@gmail.com

** Doctorado en Educación de la Universidad de Florida, de EUA. Maestría en Segunda Lenguas y Culturas en Inglés como Lengua Extranjera y Licenciatura en Lingüística Aplicada a la Enseñanza del Inglés de la Universidad Nacional, de Costa Rica. Bachillerato en la Enseñanza del Inglés y Traducción del Inglés de la Universidad Latinoamericana de Ciencia y Tecnología. Es profesora asociada de la Escuela de Idiomas y Ciencias Sociales del Instituto Tecnológico de Costa Rica. Correo:plopez.estrada@gmail.com

*** Estudiante de último año de Administración de Empresas y de segundo año de Ingeniería en Computación en el Instituto Tecnológico de Costa Rica, de Costa Rica. Es asistente de un proyecto de extensión para el fortalecimiento de las competencias del idioma inglés de una comunidad en la Región Huetar Norte, así como de un proyecto de investigación cualitativa de un estudio de caso sobre las percepciones constructivistas de mujeres empoderadas en el contexto rural comunitario. Correo: ph.estf@gmail.com 


\section{Resumen}

El curso lectivo 2020 se vio interrumpido debido a la emergencia mundial provocada por el COVID-19. En Costa Rica, el 16 de marzo del 2020, el Ministerio de Educación Pública decidió suspender la presencialidad y trabajar bajo la modalidad educativa a distancia para continuar el proceso de enseñanza-aprendizaje y evitar la desvinculación de los estudiantes del sistema educativo. Este estudio de caso, basado en una investigación cualitativa inductiva, recolectará y triangulará los datos a través de entrevistas semiestructuradas, recolección de documentación y grabaciones de videos. Se utilizará el análisis de contenido a través del uso del software WebQDA de análisis de datos cualitativos para categorizar las perspectivas de los docentes de primaria. Este estudio es una investigación en desarrollo que tiene como propósito la descripción de las percepciones de seis docentes de inglés de primaria de la Dirección Regional de Educación San Carlos con respecto de la educación a distancia para una mejor comprensión de la mediación pedagógica y del contexto profesional de los docentes durante la crisis sanitaria.

Palabras clave: COVID-19, docentes de primaria, enseñanza del inglés, investigación cualitativa.

\section{Abstract}

The 2020 academic year was interrupted due to the COVID-19 global health emergency. In Costa Rica, on March 16th, the Ministry of Public Education of Costa Rica on March 16th, 2020 decided to continue the teaching-learning process under a distant modality to avoid the students' disengagement from education. This case study, based on inductive qualitative research, will collect and triangulate data through semi-structured interviews, document gathering and video recordings, and content analysis (Hatch, 2002) to categorize the primary school teachers' perceptions through the use of WebQDA (Costa et al., 2019), a software designed to analyze qualitative data. The purpose of this proposal, that is currently under study, is to describe the perceptions of six primary school English teachers from Dirección Regional de Educación San Carlos about distance education to better comprehend the pedagogical mediation and professional context of the teachers during this health crisis.

Keywords: COVID-19, English teaching, primary teachers, qualitative research.

\section{Résumé}

L'année scolaire 202 a été interrompue par l'urgence sanitaire mondiale causée par la Covid-19. Au Costa Rica, le ministère de l'Éducation Publique annonçait le 16 mars 2020 la suspension de l'enseignement présentiel et la mise en place de la modalité à distance pour continuer le processus d'enseignement-apprentissage afin d'éviter le décrochage des élèves du système scolaire. Cette étude de cas est une recherche qualitative inductive qui vise à recueillir et trianguler les données à travers d'entretiens semidirectifs, de la collecte de documentation et de l'enregistrement des vidéos. L'analyse du contenu sera faite par le biais du logiciel WebODA pour catégoriser les perspectives des enseignants de l'école primaire. Cette recherche est en train de se développer et a pour objectif de décrire les perceptions de six enseignants d'anglais de l'école primaire du district scolaire de San Carlos au sujet de l'enseignement à distance, dans l'intention de mieux comprendre la médiation pédagogique et le contexte professionnel des enseignants pendant la crise sanitaire.

Mots-clés: Covid-19, enseignement de l'anglais, enseignants de primaire, recherche qualitative. 


\section{Introducción}

El curso lectivo 2020 se vio interrumpido de forma abrupta debido a la emergencia mundial provocada por el COVID-19. Según datos de la Organización de las Naciones Unidas para la Educación, la Ciencia y la Cultura (UNESCO), para abril, un total de 194 países habían cerrado sus sistemas educativos, afectando al $91.32 \%$ del total de la población estudiantil mundial en preescolar, primaria y secundaria ${ }^{1}$. El 16 de marzo de 2020, el Ministerio de Educación Pública (MEP) de Costa Rica tomó la decisión de suspender el curso lectivo y pasar a una modalidad de enseñanza a distancia; con lo cual, los estudiantes de primaria y secundaria asistieron únicamente 36 días a clases de forma presencial ${ }^{2}$. El propósito principal de este cambio fue continuar con el diseño e implementación de espacios de aprendizaje, replanteando la mediación pedagógica según el contexto de los estudiantes y evitar la desvinculación de los estudiantes del sistema educativo. Además, el sistema buscó establecer redes en las que los docentes pudieran apoyarse para su desarrollo profesional ${ }^{3}$.

En el documento Orientaciones para el apoyo del proceso educativo a distancia, el MEP reconoce cuatro escenarios clave para continuar con el proceso de aprendizaje: 1) estudiantes con dispositivos electrónicos y conexión a internet; 2) estudiantes con dispositivos y conexión limitada; 3) estudiantes con dispositivos sin conexión, y 4) estudiantes sin dispositivos ni conexión ${ }^{4}$. A pesar de que se pretendía regresar a la presencialidad en un período máximo de un mes, el curso lectivo se vio suspendido de manera indefinida ${ }^{5}$. Adicionalmente, una encuesta realizada a 42074 docentes en abril y mayo por el Programa del Estado de La Nación, indica que el $6 \%$ en primaria no había tenido contacto alguno con sus estudiantes, el $52 \%$ había contactado a algunos y solo el $42 \%$ había tenido contacto con todos ${ }^{6}$.

Este proceso es en esencia una modalidad de educación a distancia, en el cual existen algunos retos con respecto del contacto, acceso a internet, procesos de aprendizaje de los estudiantes y formación de los docentes en esta modalidad que llevan a la necesidad de conocer la percepción que tienen los docentes de inglés en relación con el nuevo proceso de enseñanza.

A raíz de la situación mencionada, y como parte de los procesos de colaboración interuniversitaria e interinstitucional que deben existir en el país y en las

\footnotetext{
${ }^{1}$ UNESCO, «Education: From disruption to recovery», acceso: 5 de julio de 2021, https://en.unesco.org/ covid19/educationresponse/

2 Kristin Hidalgo, «Centros educativos seguirán cerrados después de Semana Santa», 3 de marzo de 2020, Ameliarueda.com, acceso: 5 de julio de 2021, https://www.ameliarueda.com/nota/centros-educativosseguiran-cerrados-despues-de-semana-santa

3 MEP, «Orientaciones para el apoyo del proceso educativo a distancia», acceso: 5 de julio de 2020, https:// www.mep.go.cr/sites/default/files/orientaciones-proceso-educativo-distancia_0.pdf

${ }^{4}$ Ibíd.

5 Hidalgo, «Centros educativos...».

${ }^{6}$ Jennyfer León y Steffan Gómez, «¿Cómo ha sido el contacto entre docentes y estudiantes en la nueva modalidad de clases a distancia?», Blog del Estado de la Nación, 18 de agosto de 2020, https:// estadonacion.or.cr/como-ha-sido-el-contacto-entre-docentes-y-estudiantes-en-la-nueva-modalidad-de-clasesa-distancia/
} 
instituciones adscritas al Consejo Nacional de Rectores (CONARE), en junio de 2020 se inició el planteamiento de la presente propuesta de investigación por parte de dos profesores, que representan a la Universidad Estatal a Distancia (UNED), al Instituto Tecnológico de Costa Rica (ITCR) y al Ministerio de Educación Pública (MEP). La propuesta se inscribió en el Área de Gestión de Proyectos de la Escuela de Ciencias Sociales y Humanidades de la UNED. También, se estableció una red de aliados estratégicos que incluye a ambas asesoras nacionales de inglés en I y II ciclos (MEP), la actual Asesora Pedagógica de Inglés y el Jefe de Asesorías Pedagógicas de la Dirección Regional de Educación San Carlos (MEP), el coordinador de la Alianza para el Bilingüismo del Despacho Académico del Ministerio de Educación Pública (MEP), al Encargado de la Cátedra de la Enseñanza del Inglés (UNED), la Exasesora Pedagógica de Inglés de la Dirección Regional de Educación de San Carlos y actual Coordinadora de Docencia de la Universidad Técnica Nacional (UTN), y al profesor y coordinador del Área de Inglés de la Escuela de Idiomas y Ciencias Sociales del Campus Tecnológico Local San Carlos (ITCR). Los aliados forman parte de la propuesta y colaboran en proceso de realimentación, validación de los instrumentos de recolección de datos. Tomando en cuenta las diferentes áreas de conocimiento y experticia, se trabajará con ellos en procesos de validación de datos preliminares.

La presente propuesta es una investigación en desarrollo que tiene como propósito describir las percepciones de seis docentes de inglés de primaria de la Dirección Regional de Educación (DRE) de San Carlos con respecto a la educación a distancia para una comprensión de la mediación pedagógica y del contexto profesional durante la crisis sanitaria del COVID-19. Además, se plantean como objetivos específicos identificar las percepciones de seis docentes de primaria de la Dirección Regional de Educación San Carlos con respecto a la educación a distancia; contribuir con los procesos de mejora en la toma de decisiones sobre la educación pública; y fortalecer las alianzas estratégicas entre instituciones de educación pública. La finalidad de este artículo consiste en presentar la propuesta metodológica de un estudio de caso sobre la educación a distancia en tiempos de pandemia.

\section{Educación a distancia}

En la educación existen múltiples modalidades dentro de los sistemas educativos formales; una de ellas es la educación a distancia, iniciada en 1843 con cursos de taquigrafía que se dieron gracias al auge del correo postal ${ }^{7}$. En Costa Rica, la educación a distancia se remonta principalmente a 1977 con la creación de la Universidad Estatal a Distancia, la cual tenía dentro de sus funciones la promoción de la educación superior en aquellas personas que por diferentes razones no se podían integrar al sistema formal universitario ${ }^{8}$.

García-Aretio menciona, dentro de las características primordiales de la

\footnotetext{
7 Juan Chavez, Juan Martínez y Raúl Dávila, «Educación a distancia y teletrabajo», Daena: International Journal of Good Conscience 15, n. 1 (2020): 264-277, http://www.spentamexico.org/v15-n1/A19.15(1)264277.pdf

${ }^{8}$ Ley de creación de la Universidad Estatal a Distancia (UNED), Ley 6044.
} 
educación a distancia, «la no presencialidad, la comunicación no continua, el trabajo autónomo del estudiante, el trabajo fuera del aula, la utilización de recursos tecnológicos y medios técnicos, el uso de tecnologías colaborativas y la separación profesor-alumno» ${ }^{9}$. Esto indica que el estudiante debe poseer 0 desarrollar la autonomía necesaria para alcanzar los aprendizajes esperados durante su trabajo fuera de un aula, con una guía mínima por parte del docente. Además, en el proceso se utilizan recursos tecnológicos y audiovisuales que promuevan el logro de los objetivos propuestos en los planes de estudio.

Como requisitos para una educación a distancia de calidad, Holmberg ${ }^{10}$ establece siete postulados clave: primero debe existir una relación motivadora entre el docente y el estudiante que favorezca el proceso de aprendizaje; esto implica que a pesar de ser un proceso fuera del aula convencional, el docente debe buscar la forma de motivar a los estudiantes. Como segundo postulado, se presenta la autorregulación y auto instrucción que se ven directamente afectados por la realimentación del docente en cada uno de los procesos.

En tercera instancia, se propone que la realimentación adecuada genera autosatisfacción en el estudiante por los avances logrados; es decir, el docente debe ser capaz de brindar una realimentación que motive y ayude al estudiante a sentirse exitoso en sus procesos de aprendizaje. El cuarto postulado propone una relación docente-discente de armonía y parecida a la amistad; aquí, no existe tanto control por parte del docente, sino que la relación de poder debe darse de forma más horizontal que vertical.

Como quinto requisito, debe existir un proceso de diálogo para el logro de un aprendizaje significativo. El sexto postulado se refiere a la necesidad de la utilización de herramientas tecnológicas para llevar a cabo el diálogo permanente y necesario. Finalmente, como sétimo postulado, se enfatiza en la importancia de un proceso debidamente planeado y organizado para el logro de aprendizajes esperados.

Existen varias definiciones que buscan separar la educación a distancia del elearning. Aunque para García-Aretio «el e-learning o la enseñanza-aprendizaje digitales es un formato de educación a distancia basado en soportes digitales» ${ }^{11}$. Es decir, el concepto principal para establecer la educación a distancia será la separación o lejanía del docente y los discentes del espacio físico que se conoce como aula. Debido a que el MEP en sus orientaciones establece cuatro escenarios diferentes en los cuales se pueden ubicar los estudiantes, se tomará la tecnología como una herramienta más para acercar a los participantes al proceso educativo ${ }^{12}$. Sin embargo, para Hodges et al., el principal riesgo de brindar una enseñanza remota de emergencia es la confusión que puede presentarse entre los conceptos educación a distancia, enseñanza remota de

9 Yohanna Abarca, «La interacción tutor-estudiante en ámbitos de educación a distancia», Revista de Lenguas Modernas 20, (2014): 285-294.

${ }^{10}$ Abarca, «La interacción...», 294.

${ }^{11}$ Lorenzo García Aretio, «Perspectivas teóricas de la educación a distancia y virtual», Revista española de pedagogía 249, (2011): 255-271.

12 MEP, «Orientaciones para...». 
emergencia y aprendizaje virtual (e-learning). Para ellos, aunque en un periodo de emergencia se utilicen medios virtuales o a distancia, la planeación, organización y capacitación del personal docente no pueden compararse con el que se requiere para un proceso de enseñanza virtual exitoso ${ }^{13}$.

Si bien se tiene un acercamiento a la definición y características de la educación a distancia, se hace necesario definir también la educación remota de emergencia, la cual usualmente no se planea ni incluye un cambio abrupto de la forma tradicional como respuesta ante un evento. Esta situación de respuesta implica un escenario totalmente diferente a lo que se conoce como e-learning ${ }^{14}$, tal es la situación que atraviesan los sistemas educativos del mundo debido a la emergencia provocada por el COVID-19. Durante este tiempo de emergencia, Bergamaschi ${ }^{15}$ y Álvarez ${ }^{16}$ enfatizan que las medidas que se tomen desde los sistemas educativos deben garantizar el mantenimiento del vínculo existente entre los miembros de la comunidad educativa (docentes, estudiantes, familias), promover la continuidad del proceso de enseñanza mediante una puesta en práctica de los contenidos propuestos en el currículo y lograr un monitoreo efectivo de los procesos de aprendizaje.

Los diferentes países han apostado por una educación a distancia ayudada de medios «de primera generación (materiales impresos, radio y televisión) y de segunda generación (plataformas, sistemas de gestión de aprendizajes) para entregar contenido y mantener algún nivel de interacción entre escuelas y estudiantes» ${ }^{17}$. Costa Rica no es la excepción; sin embargo, entre los obstáculos que existen al impartir la educación remota de emergencia a través de medios virtuales, está la inequidad en la educación, la dificultad para obtener los materiales educativos y la queja constante de los educadores sobre la falta de compromiso y participación de los estudiantes ${ }^{18}$. Aunado a estos problemas, se puede mencionar la poca experiencia docente en ambientes de educación a distancia y la posible falta de capacitación para el uso de tecnologías.

Conociendo entonces sobre la educación remota de emergencia, y sabiendo que el MEP decidió enfocar el proceso como educación a distancia, se hace necesario

\footnotetext{
${ }^{13}$ Charles Hodges, Stephanie Moore, Barb Lockee, Torrey Trust y Aaron Bond, «The Difference Between Emergency Remote Teaching and Online Learning» (27 de Marzo, 2020). https://er.educause.edu/articles/ 2020/3/the-difference-between-emergency-remote-teaching-and-online-learning

${ }^{14}$ Zuheir Khlaif y Soheil Salha, «The Unanticipated Educational Challenges of Developing Countries in Covid19 Crisis: A Brief Report», Interdisciplinary Journal of Virtual Learning in Medical Sciences 11, n. ${ }^{\circ} 2$ (2020): 130-134. doi: 10.30476/ijvlms.2020.86119.1034

${ }^{15}$ Andrea Bergamaschi, Alejandra Cardini, Vanesa D'Alessandre, Esteban Torre y Agustina Ollivier, «Educar en pandemia: Entre el aislamiento y la distancia social», Banco Interamericano de Desarrollo, julio, 2020, doi: 10.18235/0002494

16 Horacio Álvarez, Elena Arias, Andrea Bergamaschi, Ángela López, Alessandra Noli, Marcela Ortiz, Marcelo Pérez, Sabine Rieble-Aubourg, María Rivera, Rodolfo Scannone, Madiery Vásquez y Adriana Viteri, «La educación en tiempos del coronavirus: Los sistemas educativos de América Latina y el Caribe ante COVID19», Banco Interamericano de Desarrollo, mayo, 2020, doi: 10.18235/0002337

17 Ibíd.

18 Saida Affouneh, Soheil Salha y Zuheir Khlaif, «Designing Quality E-Learning Environments for Emergency Remote Teaching in Coronavirus Crisis». Interdisciplinary Journal of Virtual Learning in Medical Sciences 11 , n. ${ }^{\circ} 2$ (2020): 135-137, doi: 10.30476/IJVLMS.2020.86120.1033
} 
establecer claramente las características que este tipo de educación debe tener. Según García-Aretio, así como múltiples autores, en la educación a distancia debe existir una separación tanto en tiempo y espacio, casi permanente, entre docente y sus estudiantes ${ }^{19}$. Esto conlleva a una predominancia del estudio independiente y autorregulación que debe poseer o desarrollar el estudiante y la necesidad de una comunicación efectiva en ambos sentidos. Esto quiere decir, que tanto el docente como el estudiante deben estar en capacidad de comunicarse entre ellos para resolver problemas sobre la marcha. Finalmente, toda institución educativa debe generar el soporte necesario para un adecuado diseño, planificación e implementación de procesos de aprendizaje centrados en el estudiante y apoyado en recursos tecnológicos de diversa índole.

La mediación pedagógica del inglés, como lengua extranjera en un contexto a distancia, varía considerablemente del mismo proceso llevado a cabo en un contexto presencial. Tal y como lo indican Roux et al., la selección del material y la forma en que se presenta a los estudiantes varía dependiendo de los valores, los recursos disponibles y el tipo de programa que se esté utilizando20. En el caso específico de Costa Rica, el MEP creó dos documentos para orientar a los docentes en este sentido: (a) Orientaciones para el apoyo del proceso educativo a distancia, y (b) Pautas para la implementación de las Guías de Trabajo Autónomo.

El primer documento indica que el docente debe crear guías de trabajo autónomo que son consideradas como «la estrategia de planificación de las actividades apoyadas con herramientas virtuales o a distancia, y que serán desarrolladas por la persona estudiante en cualquiera de los escenarios, de manera independiente o con el apoyo de algún familiar»21; es decir, el docente sustituye la mediación pedagógica usada en el aula por un documento que se hace llegar a los estudiantes de la forma que mejor convenga y tomando en cuenta el escenario en que se encuentre. Estas guías, aplicaron para todas las materias que forman parte del currículo tanto a nivel prescolar, de primaria y secundaria.

Por otro lado, en las Pautas para la implementación de las Guías de Trabajo Autónomo se desarrolla un apartado para lenguas extranjeras en el que se proponen una serie de recomendaciones a los docentes para ser tomadas en cuenta durante la elaboración e implementación de las guías en el proceso a distancia22. Dentro de las principales recomendaciones para elaborar e implementar las guías en I y II ciclos están:

\footnotetext{
${ }^{19}$ Anívar Chaves, «La educación a distancia como respuesta a las necesidades educativas del siglo XXI», Revista Academia \& Virtualidad 10, n. ${ }^{\circ} 1$ (2017): 23-41, doi: 10.18359/ravi.2241

${ }^{20}$ Ruth Roux, Nelly Trejo y Elsa González, «Distance Education for EFL Teachers: Perceptions of Learner

Support». Education and Learning Research Journal 9 (2014): 157-168, doi: 10.26817/16925777.149

${ }^{21}$ «Orientaciones ...», 15.

${ }^{22}$ MEP, «Pautas para la implementación de las Guías de Trabajo Autónomo», 2020, http://www.ddc.mep.go.cr/ sites/all/files/ddc_mep_go_cr/adjuntos/

pautas_para_la_implementacion_de_las_guias_de_trabajo_autonomo_07-05-2020vf.pdf
} 
- Las guías de trabajo deben contener tres momentos a saber: (1) me preparo para resolver la guía de trabajo autónomo, (2) voy a recordar lo aprendido y/o aprender y (3) pongo en práctica lo aprendido.

- Elaborar guías que puedan ser resueltas en un tiempo máximo de 80 minutos con un periodo intermedio de descanso.

- Redactar instrucciones claras y promover la realización de tareas lingüísticas tomando en cuenta los «Assessment Strategies» (p. 22) establecidos en los programas de estudio del 2016.

- Asegurarse de que cada actividad propuesta corresponda a una tarea lingüística específica.

- Tomar en cuenta las etapas de mediación pedagógica (warm-up, pre-task, task rehearsal, task assessment).

- Debe incluirse, en la medida de lo posible, tareas lingüísticas que promuevan las cuatro habilidades del idioma. Además, si el estudiante se encuentra en un escenario tres o cuatro, debe incorporarse el texto (script) al final de la guía para que sea posible la realización de las tareas.

- El docente debe mantener una comunicación constante y brindar realimentación oportuna del proceso de aprendizaje mediante plataformas de mensajería instantánea cuando los escenarios lo permitan.

- El proceso debe ser «gradual y progresivo y estar acorde con el nivel de dominio lingüístico de la persona estudiante» (p. 23).

- Incluir en las instrucciones el: qué hacer, cómo hacerlo y los materiales que se requieren para la ejecución de la tarea.

- Para la lengua inglesa, es importante «seguir lo sugerido en el scope and sequence, de cada semana, el cual está presente en el teacher's guide» ( $p$. $24)^{23}$.

- Redactar las instrucciones en idioma español para una mejor comprensión por parte del estudiantado y las familias.

- Finalmente, debe incluirse una sección de autoevaluación y auto regulación para que el estudiante pueda, a través de una rúbrica, indicar el nivel de aprendizaje adquirido y autoevaluar su desempeño.

${ }^{23} \mathrm{MEP}$, «Pautas para la...». 


\section{Propuesta metodológica del estudio}

El diseño de la investigación es un estudio de caso, el cual es una descripción detallada de un caso en particular y el enfoque será descriptivo ${ }^{24}$. Se escogió este tipo de estudio porque representa una sistematización en detalle de un caso con una cantidad extensiva de datos a profundidad y busca investigar sobre un fenómeno actual en un contexto real25. El caso de estudio se centrará en las percepciones de docentes de inglés de primaria que trabajan en el Ministerio de Educación Pública. El sitio de estudio será la Dirección Regional de Educación de San Carlos.

El estudio se enfoca en la investigación cualitativa inductiva, puesto que las descripciones emergerán de elementos específicos de los datos para convertirse en constructos semánticos generales, conocidos como el contenido o las categorías ${ }^{26}$. La intención de esta investigación es aprehender el significado de un fenómeno social complejo como lo es la educación a distancia en tiempos de pandemia para brindar recomendaciones en la toma de decisiones sobre la educación primaria pública.

La parte epistemológica de esta investigación se basará en el constructivismo, el cual celebra la creación del conocimiento a través de las experiencias personales e individuales ${ }^{27}$. El constructivismo toma como premisa que todas las realidades de conocimiento tienen validez y no requieren de comparaciones 0 hipótesis, ya que la realidad es un «construcción humana» y, tal cual, es única y legítima para cada individuo ${ }^{28}$. Las percepciones individuales son distintivas porque están íntimamente relacionadas con las visiones del mundo de cada individuo y su proceso de co-construcción del conocimiento en relación con otros individuos. Para este estudio, se sobreentiende que las percepciones, opiniones y experiencias de los docentes son subjetivas, pero comparten un mismo contexto educativo común en tiempos de pandemia y dentro de las directrices del MEP. Este estudio de caso busca que los docentes expongan sus experiencias y opiniones acerca del contexto educativo en el cual viven y cómo han ido forjando sus percepciones individuales dentro de este espacio colectivo de su lugar de trabajo y circunstancia sanitaria actual.

Las técnicas de recolección de datos triangulados serán entrevistas semiestructuradas, recolección de documentos y grabaciones de videos. El estudio se basará en el análisis de contenido. El análisis de contenido se basa en lecturas constantes, críticas y sistemáticas de los datos ${ }^{29}$. Se crearán códigos

\footnotetext{
${ }^{24}$ Pamela Baxter y Susan Jack, «Qualitative Case Study Methodology: Study design and implementation for novice researchers». The Qualitative Report 13, n. 4 (2008): 544-559. Recuperado de https:// nsuworks.nova.edu/tqr/vol13/iss4/2

${ }^{25}$ Robert K. Yin, Case study research and applications: Design and methods (Estados Unidos: Sage Publications, 2018).

26 J. Amos Hatch, Doing qualitative research in education settings (USA: State University of New York Press, 2002).

${ }^{27}$ Carol Grbich, Qualitative data analysis: an introduction (USA: Sage Publications, 2007).

28 Hatch, «Doing qualitative .... 15.

${ }^{29}$ Hatch, «Doing qualitative ....
} 
que resuman las unidades mínimas semánticas y que determinarán la identificación de categorías significativas para responder las preguntas de investigación del estudio:

1. ¿Qué opiniones expresan seis docentes de primaria de la Dirección Regional de Educación San Carlos con respecto a la educación a distancia llevada a cabo durante la crisis sanitaria del COVID-19?

2. ¿Cuáles son las experiencias vividas de seis docentes de primaria de la Dirección Regional de Educación San Carlos con respecto a la educación a distancia llevada a cabo durante la crisis sanitaria del COVID-19?

Este estudio de investigación intentará generar un acercamiento a la realidad que se vivió en el 2020 desde la visión de los docentes. La población meta de la investigación serán seis docentes seleccionados a través de un muestreo a propósito único con los siguientes criterios:

- Docentes de primaria que impartan lecciones tanto en I como en II ciclo.

- Docentes que laboran en escuelas donde los estudiantes se ubican en escenarios tres o cuatro propuestos por el MEP para la educación a distancia.

- Docentes en condición de propiedad.

- Docentes con grupo profesional mínimo de PT4.

- Docentes sin experiencia previa en mediación pedagógica a distancia.

- Docentes que laboren en escuelas rurales.

- Docentes que laboren en escuelas de primaria tipo Dirección 2 (con rango de matrícula total de 91-200 estudiantes y donde el director asume funciones administrativas y cuenta con un docente adicional) o tipo Dirección 3 (con rango de matrícula total 201-400 estudiantes) ${ }^{30}$.

- Docentes con una experiencia docentes entre 5 y 15 años reconocidos por el MEP.

- Tres docentes hombres y tres docentes mujeres para garantizar la paridad -la participación igualitaria de mujeres y hombres- y las cuotas de género en el estudio.

El tamaño de la muestra de seis docentes es una muestra válida y

\footnotetext{
30 MEP, «La educación subversiva: Atreverse a construir el país que queremos», 2014, https://www.mep.go.cr/ transparencia-institucional/informes-institucionales
} 
representativa dentro del enfoque de investigación cualitativa, ya que este enfoque se centra en la calidad de los datos y no en la cantidad de estos, como ocurría en una investigación cuantitativa.

El análisis de contenido se utilizará como método para identificar y analizar los patrones semánticos que van de elementos específicos a generalidades de esta investigación cualitativa ${ }^{31}$. Para este estudio, se seguirán seis fases recursivas en un proceso constante de identificación, síntesis y revisión del contenido: (1) familiarización con los datos, (2) codificación de los datos, (3) creación de categorías, (4) revisión de las categorías, (5) identificación de las categorías más sobresalientes y (6) presentación del contenido.

Para el proceso de codificación e identificación de contenido se usará WebQDA, un programa de software de análisis de datos cualitativos ${ }^{32}$. Durante los análisis preliminares y finales, se trabajará con validación interna (participantes del estudio) y validación externa (colaboradores estratégicos), como lo son la MEd. Gabriela Castillo Hernández, Exasesora Regional de Inglés de la Dirección Regional de Educación de San Carlos y actual Coordinadora Área Docencia de la Universidad Técnica Nacional, Sede Regional San Carlos; el MSc. Alexis Artavia Brenes, Jefe de Asesorías Pedagógicas y la Licda. Livia Gamboa Cordero, Asesora Pedagógica de Inglés de la Dirección Regional de Educación San Carlos; el MBA. Manuel Rojas Mata, Coordinador de Alianza para el Bilingüismo del Despacho Académico del MEP; la Dra. Ana Isabel Campos Centeno y la MEd. Yaudy Ramírez Vásquez, Asesoras Nacionales de inglés de I y II Segundo Ciclo; el MEd. Tobías Brizuela Gutiérrez, Encargado de la Catedra de Enseñanza del Inglés de la Universidad Estatal a Distancia y el Dr. Óscar Chaves Jiménez, Coordinador del Área de Inglés de la Escuela de Idiomas y Ciencias Sociales del Instituto Tecnológico de Costa Rica, Campus Tecnológico Local San Carlos. Ellos serán los colaboradores externos para la corroboración de la interpretación y presentación de los datos y garantes del rigor metodológico de la investigación.

\section{Consideraciones finales}

El sistema educativo costarricense implementó un proceso educativo a distancia como respuesta a una crisis sanitaria a nivel mundial. Es decir, por primera vez en la historia, y de forma inesperada, se pasó de una modalidad presencial a una modalidad a distancia -y en algunos casos, a un aprendizaje virtual-, lo cual implicó la creación de lineamientos, pautas y orientaciones por parte de las autoridades ministeriales en un lapso muy corto como respuesta a la problemática sanitaria.

Este estudio espera generar información pertinente que sirva de apoyo y referencia para la educación pública en la toma de decisiones debido a la probabilidad de continuar con esta metodología de enseñanza durante parte del

\footnotetext{
${ }^{31}$ Hatch, «Doing qualitative....

32 Pedro Costa, Francislê de Souza y António Moreira. WebQDA - Qualitative Data Analysis. (version 3.1) [software en línea] (2019). Aveiro University and Microio. https://www.webqda.net/
} 
curso lectivo 2021. La información que se obtenga de esta investigación será clave para el MEP, pues al ser un estudio de caso, la metodología podría ser adaptada en otros estudios en diferentes regiones del país con la finalidad de aprehender sobre la educación a distancia en tiempos de pandemia, para así tener una mejor comprensión de dicho proceso desde la perspectiva docente. Este estudio servirá como un acercamiento al sentir y pensar de los docentes como actores principales en el proceso de mediación pedagógica a distancia.

Finalmente, se espera alcanzar un fortalecimiento de las alianzas interuniversitarias e interinstitucionales entre la UNED como líder en la educación a distancia en el país, y el Campus Tecnológico Local de San Carlos del ITCR como colaborador estratega en la Región Norte del país: dos instituciones de educación superior caracterizadas por la búsqueda de la excelencia, promoviendo un trabajo en conjunto en aras de la mejora constante en la educación pública de Costa Rica.

\section{Formato de citación según APA}

Elizondo-Mejías, J., López-Estrada, P., y Pérez-Hidalgo, E. (2021). Propuesta metodológica de un estudio de caso sobre la educación a distancia en tiempos de pandemia. Revista Espiga, 20(42), 33-48.

\section{Formato de citación según Chicago-Deusto}

Elizondo-Mejías, Jonathan, López-Estrada, Patricia y Pérez-Hidalgo, Estefanía. «Propuesta metodológica de un estudio de caso sobre la educación a distancia en tiempos de pandemia». Revista Espiga 20, n. 042 (julio-diciembre, 2021): 33-48. 


\section{Referencias}

Abarca, Yohanna. «La interacción tutor-estudiante en ámbitos de educación a distancia». Revista de Lenguas Modernas 20, (2014): 285-294.

Affouneh, Saida, Soheil Salha y Zuheir Khlaif. «Designing Quality E-Learning Environments for Emergency Remote Teaching in Coronavirus Crisis». Interdisciplinary Journal of Virtual Learning in Medical Sciences 11, $\mathrm{n} . .92$ (2020): 135-137. doi: 10.30476/JVLMS.2020.86120.1033

Álvarez, Horacio, Elena Arias, Andrea Bergamaschi, Ángela López, Alessandra Noli, Marcela Ortiz, Marcelo Pérez, Sabine Rieble-Aubourg, María Rivera, Rodolfo Scannone, Madiery Vásquez y Adriana Viteri. «La educación en tiempos del coronavirus: Los sistemas educativos de América Latina y el Caribe ante COVID-19». Banco Interamericano de Desarrollo, mayo, 2020, doi: $10.18235 / 0002337$

Baxter, Pamela y Susan Jack. «Qualitative Case Study Methodology: Study design and implementation for novice researchers». The Qualitative Report 13, n. 4 (2008): 544-559. https://nsuworks.nova.edu/tqr/vol13/iss4/2

Bergamaschi, Andrea, Alejandra Cardini, Vanesa D'Alessandre, Esteban Torre y Agustina Ollivier. «Educar en pandemia: Entre el aislamiento y la distancia social». Banco Interamericano de Desarrollo, julio, 2020. doi: $10.18235 / 0002494$

Blog del Estado de la Nación. https://estadonacion.or.cr/como-ha-sido-elcontacto-entre-docentes-y-estudiantes-en-la-nueva-modalidad-de-clases-adistancia/

Chaves, Anívar. «La educación a distancia como respuesta a las necesidades educativas del siglo XXI.» Revista Academia \& Virtualidad 10, n. 1 (2017): 23-41. doi: 10.18359/ravi.2241

Chávez, Juan, Juan Martínez y Raúl Dávila. «Educación a distancia y teletrabajo». Daena: International Journal of Good Conscience 15, n.o 1 (2020): 264-277. http://www.spentamexico.org/v15-n1/A19.15(1)264-277.pdf

Costa, Pedro, Francislê de Souza y António Moreira. «WebQDA-Qualitative Data Analysis» (version 3.1) [software en línea] (2019). Aveiro University and Microio. https://www.webqda.net/ 
García-Aretio, Lorenzo. «Perspectivas teóricas de la educación a distancia y virtual». Revista española de pedagogía 249, (2011): 255-271.

Grbich, Carol. Qualitative data analysis: an introduction. Estados Unidos: Sage Publications, 2007.

Hatch, J. Amos. Doing qualitative research in education settings. Estados Unidos: State University of New York Press, 2002.

Hidalgo, Kristin. «Centros educativos seguirán cerrados después de Semana Santa», 3 de marzo de 2020, Ameliarueda.com. Acceso: 5 de julio de 2021. https://www.ameliarueda.com/nota/centros-educativos-seguirancerrados-despues-de-semana-santa

Hodges, Charles Stephanie Moore, Barb Lockee, Torrey Trust y Aaron Bond. «The Difference Between Emergency Remote Teaching and Online Learning». https://er.educause.edu/articles/2020/3/the-difference-betweenemergency-remote-teaching-and-online-learning

Khalif, Zuheir y Soheil Salha. «The Unanticipated Educational Challenges of Developing Countries in Covid-19 Crisis: A Brief Report». Interdisciplinary Journal of Virtual Learning in Medical Sciences 11, n. 2. (2020): 130-134. doi: 10.30476/ijvlms.2020.86119.1034

Ley 6044/1977, de 03 de marzo, Ley de creación de la Universidad Estatal a Distancia (UNED) (La Gaceta núm. 50 de 12 de marzo de 1977).

Ministerio de Educación Pública. «La educación subversiva: Atreverse a construir el país que queremos», 2014. https://www.mep.go.cr/transparenciainstitucional/informes-institucionales

Ministerio de Educación Pública. «Orientaciones para el apoyo del proceso educativo a distancia», 2020. https://www.mep.go.cr/sites/default/files/ orientaciones-proceso-educativo-distancia_0.pdf

Ministerio de Educación Pública. «Pautas para la implementación de las Guías de Trabajo Autónomo», 2020. http://www.ddc.mep.go.cr/sites/all/files/ ddc_mep_go_cr/adjuntos/ pautas_para_la_implementacion_de_las_guias_de_trabajo_autonomo_0705-2020vf.pdf

Roux, Ruth, Nelly Trejo y Elsa González. «Distance Education for EFL Teachers: Perceptions of Learner Support». Education and Learning Research Journal 9 (2014): 157-168. doi: 10.26817/16925777.149 
UNESCO. «Education: From disruption to recovery». Acceso: 5 de julio de 2021. https://en.unesco.org/covid19/educationresponse/

Yin, Robert K. Case study research and applications: Design and methods.

Estados Unidos: Sage Publications, 2018. 